\title{
Low simulated radiation limit for runaway greenhouse climates
}

\author{
Colin Goldblatt ${ }^{1}$, Tyler D. Robinson ${ }^{2}$, Kevin J. Zahnle ${ }^{3}$ and David Crisp ${ }^{4}$ \\ ${ }^{1}$ School of Earth and Ocean Sciences, University of Victoria, PO Box 3065, \\ Victoria, BC, V8W 3V6, Canada. \\ ${ }^{2}$ Astronomy Department, University of Washington, Box 351580, Seattle, WA \\ 98195-1580, U.S.A. \\ ${ }^{3}$ Space Science and Astrobiology Division, NASA Ames Research Center, MS \\ 245-3, Moffett Field, CA 94035, U.S.A. \\ 4 Jet Propulsion Laboratory, MS 183-501, 4800 Oak Grove Drive, Pasadena, CA \\ 91109, U.S.A.
}

\begin{abstract}
Terrestrial planet atmospheres must be in long-term radiation balance, with solar radiation absorbed matched by thermal radiation emitted. For hot moist atmospheres, however, there is an upper limit on the thermal emission which is decoupled from the surface temperature. If net absorbed solar radiation exceeds this limit the planet will heat uncontrollably, the so-called "runaway greenhouse". Here we show that a runaway greenhouse induced steam atmosphere may be a stable state for a planet with the same amount of incident solar radiation as Earth has today, contrary to previous results. We have calculated the clear-sky radiation limits at line-by-line spectral resolution for the first time. The thermal radiation limit is lower than previously reported $\left(282 \mathrm{~W} \mathrm{~m}^{-2}\right.$ rather than $\left.310 \mathrm{~W} \mathrm{~m}^{-2}\right)$ and much more solar radiation would be absorbed $\left(294 \mathrm{~W} \mathrm{~m}^{-2}\right.$ rather than $\left.222 \mathrm{~W} \mathrm{~m}^{-2}\right)$.
\end{abstract}


Avoiding a runaway greenhouse under the present solar constant requires that the atmosphere is subsaturated with water, and that cloud albedo forcing exceeds cloud greenhouse forcing. Greenhouse warming could in theory trigger a runaway greenhouse but palaeoclimate comparisons suggest that foreseeable increases in greenhouse gases will be insufficient to do this.

Earth's surface and troposphere are heated by the Sun. If more sunlight was absorbed, these would warm and, consequently, emit more thermal radiation to space. Whilst thermal radiation from the surface can escape the atmosphere directly, energy balance and temperate climate are maintained. Most such emission occurs through the "water vapour window", the 8 to $13 \mu \mathrm{m}$ local minimum in water vapour absorption, coincident with the Wein peak in surface thermal emission at $10 \mu \mathrm{m}$.

Warming an Earth-like planet would make the atmosphere moist and hence optically thick across the thermal region, even in the water vapour window. Then, only the upper troposphere (not the surface or lower troposphere) would be able emit radiation directly to space. As sunlight penetrates the atmosphere better than thermal infrared radiation, the upper troposphere is heated from below and its thermal structure determined by moist convection. The water vapour mixing ratio increases with temperature, causing the moist adiabatic lapse rate to tend towards the saturation vapour pressure curve and the tropopause to acquire a fixed temperature-pressure structure. Thus the level from which effective thermal emission occurs tends to a fixed temperature and the flux a fixed value. This maximum in outgoing radiation - or "radiation limit" - means that surface warming no longer leads 
to more thermal emission. If the net absorption of solar radiation exceeds this limit, then surface temperatures will increase in a "runaway greenhouse", evaporating the entire ocean and sterilising the planet en route.

This radiation limit was first found in a model by Simpson in 1927 (ref. [1]), but seen as a paradox. The physics, as described above, was subsequently elucidated by Nakajima et al.[2], so we refer to the SimpsonNakajima limit. In the intervening time, the notion of a runaway greenhouse was identified by reference to a separate stratospheric radiation limit, the Komabayashi-Ingersoll limit[3, 4] (which is unlikely to be reached in practice[2]). These various radiation limits have recently been reviewed[5]. Numerical calculations performed a few decades ago by several groups $[6$, $7,8,9]$ converged on a consensus estimate of $310 \mathrm{~W} \mathrm{~m}^{-2}$ for the SimpsonNakajima limit[9]. Subsequent work addressed some aspects of the problem (e.g. the hydrological cycle[10], pedagogic treatment of thermal limits[11], early Mars[12]), but there has been no full revision of the classic calculations.

Here, we present the most complete study of the runaway greenhouse for 25 years, across the full range of temperatures, using modern input spectroscopic data and a line-by-line treatment of the solar and thermal radiation (see the Methods section). We limit ourselves to clear-sky (cloud-free) calculations, which embody the first-order physics of the problem. Clouds both reflect solar radiation (making the runaway less likely) and enhance the greenhouse (making it more likely). Omitting them yields hard upper bounds on both solar absorption and thermal emission. This is a robust place to begin a re-evaluation of the problem, with thought experiments on how clouds will modify the results. 
The runaway greenhouse has contemporary relevance. There has been high profile speculation that extreme anthropogenic global change could trigger it[13], but this is contrary to existing theory[2, 5] and numerical results[14]. It also sets the inner boundary of the circumstellar "habitable zone" $[15,16]$ in which the Kepler mission is presently identifying planetary candidates[17].

\section{Pure water atmospheres}

We begin with the absolute end member case of a pure water atmosphere: neither any background gas nor any greenhouse gas other than water. With increasing surface temperature, evaporation from the ocean adds mass to the bottom of the atmosphere, so this is a good approximation for hot atmospheres in which the water vapour mixing ratio asymptotes to one.

The optical depth of the atmosphere, $\tau_{\lambda}$ (a function of wavelength), is measured downward from the top. Effective emission to space and attenuation of sunlight occur where $\tau_{\lambda} \sim 1$ (given Beer's law; $I_{1}=I_{0} \exp ^{-\tau_{\lambda}}$, where $I_{0}$ and $I_{1}$ are the incident and transmitted radiance), so plotting the altitude of $\tau_{\lambda}=1$ shows where emission to space and absorption of sunlight dominantly occur (Fig. 1)

For thermal emission, $\tau_{\lambda}=1$ is either near the surface or not reached for low surface temperatures $\left(T_{s}\right)$, but "lifts off" towards high altitudes as the planet warms. Whilst $T_{s} \lesssim 1600 \mathrm{~K}$, the temperature of $\tau_{\lambda}=1$ remains between 250 and $300 \mathrm{~K}$, so the top of atmosphere thermal spectrum is bounded by Planck functions for temperatures of 250 and $300 \mathrm{~K}$, and is independent 
of $T_{s}$. Thus the Simpson-Nakajima radiation limit emerges as $282 \mathrm{~W} \mathrm{~m}^{-2}$ (Fig. 2), lower than previous estimates (e.g. $310 \mathrm{~W} \mathrm{~m}^{-2}$, ref [9]). When $T_{s} \gtrsim 1600 \mathrm{~K}$ the upper atmosphere temperature gradient is sufficiently steep that the temperature reaches $400 \mathrm{~K}$ at $\tau_{\lambda}=1$ in the $4 \mu \mathrm{m}$ water vapour window (Fig. 1c). Hence a new peak in thermal radiation emerges, sufficient to permit a new stable climate with a steam atmosphere. No surface radiation escapes directly to space. Observation of this emission peak in a (exo)planetary atmosphere would indicate that the planet is in a runaway greenhouse state.

Earth's atmosphere is largely transparent to solar radiation. However, for water rich atmospheres, increasing temperatures are accompanied by increases in atmosphere pressure and water vapour absorption. The additional pressure increases the Rayleigh scattering optical depths at shorter wavelengths, while near infrared water vapour vibration rotation bands increase the absorption optical depth at longer solar wavelengths. Both processes attenuate sunlight, very little of which reaches the surface, so surface albedo no longer affects the radiation budget. In the limiting case, for a pure water atmosphere without clouds and the present solar flux, a maximum of $294 \mathrm{~W} \mathrm{~m}^{-2}$ is absorbed, much higher than the previous estimate[9] of $222 \mathrm{~W} \mathrm{~m}^{-2}$.

Given a hot, moist and cloud free atmosphere, the net absorption of sunlight would slightly exceed the thermal radiation limit. This imples that a cloudless runaway greenhouse, steam atmosphere, would be stable under the present insolation. Earth today has a stable temperate climate (the requirements for which are discussed in the next section) implying a climate bistability with respect to the runaway greenhouse (previously seen 
in a grey atmosphere model[18]). Both the solar and thermal calculations represent upper bounds for a pure water atmosphere - clouds could reduce either, moving the bifurcation point.

Previous work suggested that the thermal radiation limit does not depend on presence of non-condensible greenhouse gases $[9,2]$. This is not strictly correct. The radiation limit depends on the minimum absorption cross section in the $10 \mu \mathrm{m}$ water vapour window; any additional opacity here would raise the $\tau_{\lambda}=1$ surface to a higher altitude that radiates at a lower temperature. As purely theoretical tests, we set $1 \%$ each of our atmospheres to be carbon dioxide or ammonia, then $1 \%$ of both. The radiation limit decreased by $2 \mathrm{~W} \mathrm{~m}^{-2}$ for $1 \% \mathrm{CO}_{2}, 6 \mathrm{~W} \mathrm{~m}^{-2}$ for $1 \% \mathrm{NH}_{3}$ and $8 \mathrm{~W} \mathrm{~m}^{-2}$ for both (supplementary figure 7). Ammonia is one of the strongest absorbers around $10 \mu \mathrm{m}$, so deeper reductions to the radiation limit seem unlikely.

These results are sensitive to the absorption cross sections used. Firstly, using the most detailed spectral line-list for water (we use HITEMP2010[19]) and correct Rayleigh scattering cross sections for water are essential. Using a less comprehensive line list (e.g. HITRAN 2008), or Rayleigh scattering for air instead of water, gives erroneous results (supplementary figures 36). Relative to previous results, our lower thermal emission and higher solar absorption are due to these absorption coefficient changes and increased spectral resolution. Secondly, the strength of the water vapour continuum (the smoothy varying absorption in the window regions) is very important. In the infrared, the continuum we use is weaker than indicated by the most recent data[20] (supplementary figure 9), so our estimates are conservative and the Simpson-Nakajima limit is likely somewhat lower than our estimate. 
The uncertainty wedge associated with the continuum grows towards shorter wavelengths, and there are no measurements in the visible region. Our (or any other) solar calculations must be regarded as provisional until such measurements are made.

\section{Transition to a runaway greenhouse}

Given that a cloud-free steam atmosphere is a stable state at present solar constant, one should examine both how the stable temperate climate is maintained on Earth and the conditions which would lead to a runaway greenhouse. Hence we examined transitional atmospheres (up to $400 \mathrm{~K}$ ) with the same mass of background gas as Earth and various greenhouse gas inventories (Table 1$)$.

At $280 \mathrm{~K}$, the surface emits directly to space through the water vapour window (Figure 3). For surface temperatures above $310 \mathrm{~K}$ the temperature of the emitting level remains between 250 to $300 \mathrm{~K}$, regardless of the surface temperature. If greenhouse gases other than water are more abundant, $\tau_{\lambda}=1$ is higher in the absorption bands of these gases and less radiation is emitted overall. However, the relative magnitude of this effect decreases in hotter atmospheres with more water. In flux terms (Figure 4), for the end-member case of a saturated, cloud free atmosphere with contemporary surface albedo, the net absorbed solar radiation exceeds thermal emission in all scenarios except that with no greenhouse gases other than water, implying that a runaway greenhouse should occur. As this has manifestly not happened to Earth, we are led to the conclusion that a combination of atmospheric 
subsaturation and an excess of cloud albedo forcing over cloud greenhouse forcing prevents a runaway greenhouse on Earth today.

First, we relax the assumption of saturation. Our nominal relative humidity profile (see Methods) yields a stable climate only for the case of no additional greenhouse gases, although a marginally stable result was obtained for pre-industrial greenhouse gas concentrations. The assumed relative humidity profile is a source of uncertainty in 1-D models. With convection parameterisation in a 1-D model, Rennó[10] found a non-linear transition from sub-saturation to saturation around $310 \mathrm{~K}$, introducing an additional bistability in climate which is not found with climatological relative humidity profiles like ours[10]. In Earth's tropics, columns of dry air prevent a local runaway greenhouse[21]. Nonetheless, our results indicate that subsaturation alone is probably not sufficient to prevent a runaway greenhouse today (Fig. $4)$.

Thus, we turn our attention to clouds. Today, these give an albedo forcing of $50 \mathrm{~W} \mathrm{~m}^{-2}$ and a greenhouse forcing of $26 \mathrm{~W} \mathrm{~m}^{-2}$ (ref [22]). Climatological mean top of atmosphere fluxes are $239.4 \mathrm{~W} \mathrm{~m}^{-2}$ net solar absorbed and $238.5 \mathrm{~W} \mathrm{~m}^{-2}$ outgoing thermal[23] (the $0.9 \mathrm{~W} \mathrm{~m}^{-2}$ discrepancy is causing global warming). High, cold, clouds have a dominant greenhouse effect (though also reflect). Low cloud has a dominant albedo effect. At first approximation, we simulate this by increasing surface albedo; doubling our $12 \%$ albedo approximately represents the low cloud forcing. Our subsaturated preindustrial cases with present surface temperature and enhanced albedo (figure 4) have TOA solar and thermal fluxes of 263 and $266 \mathrm{~W} \mathrm{~m}^{-2}$, reasonably approximating climatology minus high cloud forcing. In all our 
scenarios (other than arbitrarily high $\mathrm{CO}_{2}$ ), there is a stable climate under this assumption. Thus we can infer that the excess of cloud albedo over cloud greenhouse forcing, in combination with subsaturation, permits stable temperate climate on Earth.

The clear sky fluxes are upper bounds: clouds could reduce either flux, making the runaway more or less likely. Previously[9] it was argued that cloud reflection would dominate over cloud greenhouse in an optically thick atmosphere. However, this misses a critical distinction based on the level of the clouds. The atmosphere is more transparent to sunlight than thermal radiation, so $\tau_{\lambda}($ solar $)=1$ is at lower altitude than $\tau_{\lambda}($ thermal $)=1$, so there are three categories of cloud effect based on these. Below $\tau_{\lambda}($ solar $)=1$, clouds will have negligible effect. Between $\tau_{\lambda}($ solar $)=1$ and $\tau_{\lambda}($ thermal $)=1$, albedo will dominate. Above $\tau_{\lambda}$ (thermal) $=1$, cloud greenhouse will likely dominate (though reflection may dominate if the clouds are more than four times thicker than the present global mean[24]). The largest projected increase in the water vapour mixing ratio is in the upper atmosphere (supplementary figure 1), suggesting that high clouds would increase most (though this is speculative). For near future global warming, the current best estimates are for clouds to exert a positive forcing[25] (i.e. enhanced greenhouse dominating).

Steady state climates exist where the net flux (thermal minus solar) is zero; stable where the net flux increases with temperature and unstable where it decreases with temperature (Fig. 4). For small greenhouse gas inventories, the outgoing thermal flux overshoots the Simpson-Nakajima limit giving a "hump of stability: excess thermal emission will give a negative feedback, 
restoring stable, temperature climate. Using the subsaturated, $25 \%$ albedo runs as a reference, the "hump of stability" is $24 \mathrm{~W} \mathrm{~m}^{-2}$ for preindustrial, $18 \mathrm{~W} \mathrm{~m}^{-2}$ for RCP 8.5 at 2100 and $8 \mathrm{~W} \mathrm{~m}^{-2}$ for extreme anthropogenic. With our arbitrarily high greenhouse gas scenario $\left(30,000\right.$ ppmv $\mathrm{CO}_{2}$, this vanishes and there is no stable temperate climate. Greenhouse gases do not simply warm the planet, but also lower or remove the energy barrier between temperate climate and a runaway greenhouse.

\section{Other times and other planets}

A runaway greenhouse has manifestly not occurred on post-Hadean Earthit would have sterilised Earth (there is observer bias). Palaeoclimate gives us a sample of conditions where a runaway greenhouse did not occur, but cannot tell us the size of any safety margin. The "hothouse" climate of the Eocene is the most useful constraint for anthropogenic change. With solar constant $1 \%$ less than today and a few thousand ppmv $\mathrm{CO}_{2}$, the mean temperature was $\sim 10 \mathrm{~K}$ warmer than today[26]. With $\mathrm{CO}_{2}$ and temperature both higher then than we expect in the foreseeable future [27], this implies that an anthropogenic runaway greenhouse is unlikely. Deglaciaton from Neoproterozoic "snowball Earth" events likely required that $\sim 10 \%$ of the atmosphere was carbon dioxide. The solar constant was $6 \%$ less than today, so net solar radiation absorbed would have been $12 \mathrm{~W} \mathrm{~m}^{-2}$ less and climate not yet bistable. By contrast, deglaciation from the next Snowball Earth might trigger a runaway.

Venus probably experienced a runaway greenhouse in the past, evident 
now in enrichment of $\mathrm{D} / \mathrm{H}$ in its atmosphere[28, 7]. Previous work suggested that early Venus was close to the threshold for a runaway greenhouse[9] — our new lower radiation limit and enhanced solar absorption imply that, given the same amount of nitrogen in the atmosphere as Earth, Venus may not have had a habitable period. However, if early Venus had at least as much nitrogen in its atmosphere as it does now this would have had a protective effect (Figure 5). More nitrogen gives more Rayleigh scattering, decreasing absorbed solar radiation in a transitional atmosphere.

As the solar constant increases with time, Earth's future is analogous to Venus's past. We expect a runaway greenhouse on Earth 1.5 billion years hence if water is the only greenhouse gas, or sooner if there are others. Earth's atmospheric nitrogen inventory has likely changed with time[29]. Any future decrease would lessen the protective effect of Rayleigh scattering and hasten a runaway greenhouse (somewhat by contrast to previous arguments[30]).

Our pure water calculations were aimed at hot atmospheres, but should also apply to "water-worlds", analogous to a warm version of Jupiter's moon Europa. In the absence of Rayleigh scattering from background gas, planetary albedo would be lower than Earth. Under Earth's insolation, without clouds and with $12 \%$ surface albedo (the average for Earth, about twice that of seawater), there is no stable temperate climate and runaway greenhouse would always ensue. Arbitrarily increasing surface albedo to 25\% (a proxy for low cloud reflection) gives a marginally stable state at $275 \mathrm{~K}$. With a mean surface temperature this low, ice albedo feedback would likely lead to low latitude glaciation (Snowball Earth-like). A transient warming sufficient to melt the ice would likely cause a transition directly to a runaway green- 
house. Unless mediated by other atmospheric constituents or clouds, there would be no stable temperate climate state.

The runaway greenhouse sets a hard limit for the inner edge of the circumstellar "habitable zone" $[15,16]$. This classic definition neglects multiple climate equilibria so may be misleading. Three major stable climate states exist at the same solar constant: Snowball Earth (at least transiently reduced habitability), temperate (habitable) or runaway greenhouse steam atmosphere (sterilizing)[18]. Changes to clouds today may be sufficient to transition from temperate to either other state. Determining surface temperature requires knowledge of the atmospheric state and history: it is not possible to determine a habitability a priori from incident stellar radiation.

The thermal radiation limit depends weakly on the mass of the planet (Supplementary figure 8). Everything else being equal, a Mars size planet would be more susceptible to a runaway greenhouse and a so-called "superEarth" less[11].

Revisiting the classic planetary sciences problem of the runaway greenhouse with modern modelling tools, we have shown that the thermal radiation limit is lower and that more solar radiation is absorbed. The runaway greenhouse may be much easier to initiate than previously thought. A renewed modelling effort is needed, addressing both Earth and planetary science applications. We have begun this process with a single column, clear-sky model, which has allowed us to advance the core radiative transfer aspect of the problem. Reference calculations are available as Supplementary Information to permit future model testing. Our work should be followed with cloudy column models then, ultimately, general circulation models (to address the 
cloud and relative humidity distributions). The latter represents a "grand challenge" in climate modelling, for which present-generation models may be insufficient: there are difficulties associated with radiative transfer, clouds and dynamics (with a major component being condensible), and no empirical comparison cases. 


\section{Methods}

\section{Atmospheric structure}

We prescribe the surface temperature and atmospheric structure (moist adiabatic, with bottom of atmosphere temperature equal to surface temperature) in a single global mean column[9]. We separately consider pure water atmospheres (which is the limit for extensive evaporation of the ocean and allows us to easily include the non-ideal behaviour of steam) and transitional atmospheres (up to a surface temperature of $400 \mathrm{~K}$ where the ideal gas law remains a good assumption). More information is given in the supplementary information, along with profiles (supplementary figure 1).

\section{Relative humidity}

The standard assumption for one-dimensional models is a linear decrease in relative humidity with pressure, from the surface to the tropopause[31, 32]. Mean subsaturation on Earth today is an area-weighted construction of areas of saturated and unsaturated air. Once water vapour is a major atmospheric constituent (a few percent or more), regions of sub saturation would induce huge pressure gradients, leading to rapid mixing from saturated regions and permitting further evaporation. Hence we assume that air below a level with some threshold water vapour mixing ratio (we use 5\%) is saturated, with a linear decrease in relative humidity with pressure above. 


\section{Spectral data}

We use line data for water from the HITEMP2010 spectral database[19], which provides line data, including weak lines, in the range $0-30,000 \mathrm{~cm}^{-1}$ ( $>0.33 \mu \mathrm{m}$; Supplementary figure 3 ) and absorption cross sections for the far $\mathrm{UV}\left(>50,000 \mathrm{~cm}^{-1},<0.2 \mu \mathrm{m}\right)$. The line data is converted to cross sections separately for each atmospheric profile examined using the LBLABC program, written by David Crisp. There is no spectral data available in the mid UV $\left(30000-50000 \mathrm{~cm}^{-1}, 0.2-0.33 \mu \mathrm{m}\right)$. This corresponds to $6 \%$ of incoming solar radiation, so we will underestimate net absorption. It is of utmost importance to use the most detailed line database available for water (supplementary figures 3-4). For thermal emission, HITRAN2004 and later perform similarly to HITEMP2010 up to $625 \mathrm{~K}$ whereas HITRAN2k allows more outgoing radiation through the 4 and $10 \mu \mathrm{m}$ windows. For solar radiation, all HITRAN datasets give less absorption than HITEMP2010. At high temperatures (thick atmospheres) the largest differences are seen at shorter wavelengths as net absorption depends on the ratio of absorption to Rayleigh scattering cross sections (the latter depending on the reciprocal of the fourth power of wavelength). For greenhouse gases other than water, we use line data from HITRAN2008[33].

\section{Continuum absorption}

The water vapour continuum is smoothy varying absorption which cannot be explained by near-centre contributions of known spectral lines. It is primarily responsible for setting the absorption cross section in the water vapour 
window, which in turn largely determines the radiation limit. There has been interesting theoretical work on the source of this recently[34], but it will be some time until this provides a mature parameterisation at the range of temperature, pressure and mixing ratio conditions that we encounter. Our approach is to use a more standard implementation, which is implemented with $\chi$-factors (ref. [35] and Clough, personal communication, 2000) and performs similarly in tests to the empirical MT-CKD 2.4 continuum. We then test our computed absorption cross sections against the best available data in the thermal infrared[20] (supplementary figure 9). Our implementation somewhat underestimates the strength of the continuum, so the radiation limit may be lower than our estimate and thermal exit from the runaway would require a warmer temperature. Whilst the temperature-pressure range of the data is limited, we take comfort in that the thermal radiation limit is determined in the cool upper atmosphere, which is within observable ranges.

\section{Rayleigh scattering}

Previous work[9] used Rayleigh scattering cross sections for air throughout, as data for water was not available (J. Kasting, personal communication). Detailed refractive indices for water are now available[36], so we derive new constants for Rayleigh scattering (supplementary table 1). Water is a weaker Rayleigh scatterer than air, so using Rayleigh scattering cross sections for air in place of water leads to erroneously low solar absorption, as too much sunlight is scattered (supplementary figure 6). 


\section{Radiative transfer calculation}

We use the SMART code, written by David Crisp[37], for our radiative transfer calculations. This code works at line-by-line resolution, but uses a spectral mapping algorithm to treat different wavenumber regions with similar optical properties together, giving significant savings in computational cost. We

evaluate the radiative transfer in the range $50-100,000 \mathrm{~cm}^{-1}(0.1-200 \mu \mathrm{m})$ as a combined solar and thermal calculation. Our solar source is spectrally resolved and we average the flux from zenith angles of $7.5^{\circ}, 22.5^{\circ}, 37.5^{\circ}, 52.5^{\circ}$, $67.5^{\circ}$ and $82.5^{\circ}$. In a few cases, models for one of these zenith angles did not run successfully due to singularities in the matrix inversion in DISORT[38], which SMART uses for multiple scattering. In these cases, an appropriate average was made of the remaining five and the effect on the final results is trivial. Spectra displayed are reduced via a simulated slit function. Our reference-standard radiative transfer model output is available as supplementary information to facilitate future model testing. 


\section{References}

[1] Simpson, G. C. Some studies in terrestrial radiation. Mem. Roy. Met. Soc. 11, 69-95 (1927).

[2] Nakajima, S., Hayashi, Y.-Y. \& Abe, Y. A study of the "runaway greenhouse effect" with a one-dimensional radiative-convective model. J. Atmos. Sci. 49, 2256-2266 (1992).

[3] Komabayashi, M. Discrete equilibrium temperatures of a hypothetical planet with the atmosphere and the hydrosphere of a one componenttwo phase system under constant solar radiation. J. Meteor. Soc. Japan 45, 137-139 (1967).

[4] Ingersoll, A. P. The runaway greenhouse: a history of water on Venus. J. Atmos. Sci. 26, 1191-1198 (1969).

[5] Goldblatt, C. \& Watson, A. J. The runaway greenhouse: implications for future climate change, geoengineering and planetary atmospheres. Phil. Trans. 370, 4197-4216 (2012).

[6] Pollack, J. B. A Nongrey Calculation of the Runaway Greenhouse: Implications for Venus' Past and Present. Icarus 14, 295-306 (1971).

[7] Watson, A. J., Donahue, T. M. \& Kuhn, W. R. Temperatures in a runaway greenhouse on the evolving Venus Implications for water loss. Earth Plan. Sci. Lett. 68, 1-6 (1984). 
[8] Abe, Y. \& Matsui, T. Evolution of an impact generated $\mathrm{H}_{2} \mathrm{O}-\mathrm{CO}_{2}$ atmosphere and formation of a hot proto-ocean on Earth. J. Atmos. Sci. 45, 3081-3101 (1988).

[9] Kasting, J. F. Runaway and moist greenhouse atmospheres and the evolution of Earth and Venus. Icarus 74, 472-494 (1988).

[10] Rennó, N. O. Multiple equilibria in radiative-convective atmospheres. Tellus A 49, 423-438 (1997).

[11] Pierrehumbert, R. T. Principles of planetary climate (Cambridge University Press, Cambridge, UK, 2010). Pp. 652.

[12] Segura, T., Mckay, C. \& Toon, O. An impact-induced, stable, runaway climate on mars. Icarus 220, 144-148 (2012).

[13] Hansen, J. Storms of My Grandchildren: The Truth About the Coming Climate Catastrophe and Our Last Chance to Save Humanity (Bloomsbury, New York, 2009).

[14] Kasting, J. F. \& Ackerman, T. P. Climatic consequences of very highcarbon dioxide levels in the Earth's early atmosphere. Science 234, $1383-1385$ (1986).

[15] Kasting, J. F., Whitmere, D. P. \& Reynolds, R. T. Habitable zones around main sequence stars. Icarus 101, 108-128 (1993).

[16] Abe, Y. Physical state of the very early Earth. Lithos 30, 223-235 (1993). 
[17] Batalha, N. M. et al. Planetary Candidates Observed by Kepler, III: Analysis of the First 16 Months of Data. Astrophys. J. Supp. 204, arXiv:1202.5852 (2013).

[18] Ishiwatari, M., Nakajima, K., Takehiro, S. \& Hayashi, Y.-Y. Dependence of climate states of gray atmosphere on solar constant: From the runaway greenhouse to the snowball states. J. Geophys. Res. 112, D13120 (2007).

[19] Rothman, L. S. et al. HITEMP, the high-temperature molecular spectroscopic database. J. Quant. Spect. Ra. Tran. 111, 2139-2150 (2010).

[20] Baranov, Y., Lafferty, W., Ma, Q. \& Tipping, R. Water-vapor continuum absorption in the $800-1250 \mathrm{~cm}^{-1}$ spectral region at temperatures from 311 to 363 K. J. Quant. Spect. Ra. Tran. 109, 2291-2302 (2008).

[21] Pierrehumbert, R. T. Thermostats, radiator fins and the local runaway greenhouse. J. Atmos. Sci. 52, 1784-1806 (1995).

[22] Zhang, Y. C., Rossow, W. B., Lacis, A. A., Oinas, V. \& Mishchenko, M. I. Calculation of radiative fluxes from the surface to top of atmosphere based on ISCCP and other global data sets: Refinements of the radiative transfer model and the input data. J. Geophys. Res. 109, D19105 (2004).

[23] Trenberth, K. E., Fasullo, J. T. \& Kiehl, J. T. Earth's global energy budget. Bull. Amer. Meteor. Soc. 90, 311-324 (2009). 
[24] Goldblatt, C. \& Zahnle, K. J. Clouds and the faint young sun paradox. Clim. Past 7, 203-220 (2011).

[25] Soden, B. \& Held, I. An assessment of climate feedbacks in coupled ocean-atmosphere models. J. Climate 19, 3354-3360 (2006).

[26] Zachos, J., Pagani, M., Sloan, L., Thomas, E. \& Billups, K. Trends, Rhythms, and Aberrations in Global Climate 65 Ma to Present. Science 292, 686-693 (2001).

[27] Archer, D. \& Brovkin, V. The millennial atmospheric lifetime of anthropogenic $\mathrm{CO}_{2}$. Climatic Change 90, 283-297 (2008).

[28] Donahue, T. M., Hoffman, J. H., Hodges, R. R. \& Watson, A. J. Venus was wet - a measurement of the ratio of deuterium to hydrogen. Science 216, 630-633 (1982).

[29] Goldblatt, C. et al. Nitrogen-enhanced greenhouse warming on early earth. Nature Geosci. 2, 891-896 (2009).

[30] Li, K.-F., Pahlevan, K., Kirschvink, J. L. \& Yung, Y. L. Atmospheric pressure as a natural climate regulator for a terrestrial planet with a biosphere. P. Nat. Acad. Sci. USA 106, 9576-9579 (2009).

[31] Manabe, S. \& Wetherald, R. D. Thermal equilibrium of the atmosphere with a given distribution of relative humidity. J. Atmos. Sci. 24, 241259 (1967).

[32] Forster, P. M. d. F., Freckleton, R. S. \& Shine, K. P. On aspects of the concept of radiative forcing. Clim. Dynam. 13, 547-560 (1997). 
[33] Rothman, L. S. et al. The HITRAN 2008 molecular spectroscopic database. J. Quant. Spectrosc. Ra. Tran. 110, 533-572 (2009).

[34] Ptashnik, I. V., Shine, K. P. \& Vigasin, A. A. Water vapour selfcontinuum and water dimers: 1. Analysis of recent work. J. Quant. Spect. Ra. Tran. 112, 1286-1303 (2011).

[35] Clough, S., Kneizys, F. \& Davies, R. R. Line shape and the water vapor continuum. Atmos. Res. 23, 229-241 (1989).

[36] Harvey, A. H., Gallagher, J. S. \& Leverlt Sengers, J. M. H. Revised formulation for the refrractive indes of water and steam as a function of wavelength, temperature and desnity. J. Phys. Chem. Ref. Data 27, 761-774 (1998).

[37] Meadows, V. S. \& Crisp, D. Ground-based near-infrared observations of the Venus nightside: the thermal structure and water abundance near the surface. J. Geophys. Res. 101, 4595-4622 (1996).

[38] Stamnes, K., Tsay, S.-C., Wiscombe, W. \& Jayaweera, K. Numerically stable algorithm for discreete-ordinate-method radiative transfer in multiple scattering and emmitting layered media. Appl. Optics. 27, 2502-2509 (1988).

[39] Collins, W. D. et al. Radiative forcing by well-mixed greenhouse gases: Estimates from climate models in the Intergovernmental Panel on Climate Change (IPCC) Fourth Assessment Report (AR4). J. Geophys. Res. 111, D14317 (2006). 
[40] R. Moss et. al. Towards new scenarios for analysis of emissions, climate change, impacts, and response strategies. Tech. Rep., IPCC, Geneva (2008). 
Supplementary Information is linked to the online version of the paper at www.nature.com/ngeo.

Acknowledgements: We thank David Catling, Jim Kasting, Ray Pierrehumbert and Andy Watson for discussions at various stages in the project, and Dorian Abbot for a constructive review. Contributions to this work were funded by NASA Planetary Atmospheres and NSERC Discovery grants awarded to C.G. and by the NASA Astrobiology Institute Virtual Planetary Laboratory.

Author Contributions: C.G. and K.J.Z. suggested the study. C.G. conducted all of the modelling work, using a model written by D.C., with help from T.R. and D.C. All authors contributed to analyzing the results.

Author Information: Reprints and permissions information is available at www.nature.com/reprints. The authors declare no competing financial interests. Correspondence and requests for materials should be addressed to C.G.: czg@uvic.ca. 


\begin{tabular}{lccc}
\hline Scenario & $\mathrm{CO}_{2}(\mathrm{ppmv})$ & $\mathrm{CH}_{4}(\mathrm{ppmv})$ & $\mathrm{N}_{2} \mathrm{O}(\mathrm{ppbv})$ \\
\hline Baseline & 0 & 0 & 0 \\
Preindustrial & 287 & 0.806 & 275 \\
RCP 8.5, year 2100 & 936 & 3.75 & 435 \\
Extreme anthropogenic & 3000 & 10 & 500 \\
Arbitrarily high & 30,000 & 10 & 500 \\
\hline
\end{tabular}

Table 1: Greenhouse gas inventory scenarios used in transitional atmospheres. Preindustrial[39] and RCP (representative concentration pathway) 8.5 for year 2100 (ref [40]) are standard. Extreme anthropogenic assumes that the large fossil fuel reservoirs (coal and some methane clathrate) are burned rapidly - i.e. it is extreme, but certainly possible. Note that concentrations are relative to a standard, $10^{5} \mathrm{~Pa}$ atmosphere and the mass of each greenhouse gas is fixed as temperature and surface pressure increase, so actual mixing ratios decrease as temperature increases. 

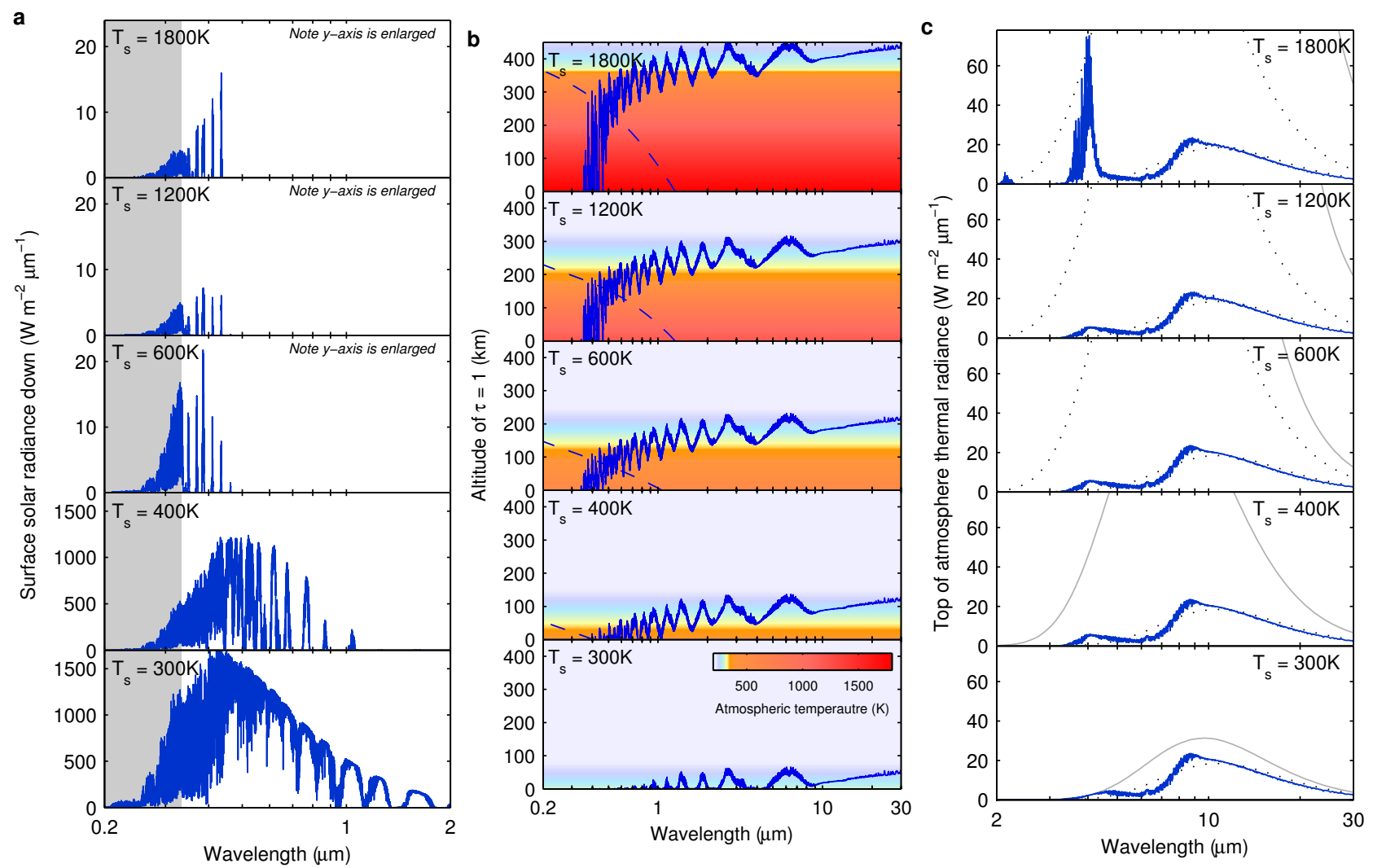

Figure 1: Spectra and effective absorption/emission levels in a pure water atmosphere $T_{s}$ is surface temperature. a. Spectrum of downward solar flux at the surface. No line data is available for the grey shaded area. $\mathbf{b}$. Altitude at which optical depth is unity. Solid line is absorption optical depth and dashed line is Rayleigh scattering optical depth. Background shading is atmospheric temperature. c. Spectrum of outgoing thermal flux at the top of the atmosphere. Solid lines are the black body flux at the surface temperature. Dotted lines are the black body flux for $280 \mathrm{~K}$ and $400 \mathrm{~K}$ for comparison. See also supplementary figure 2 

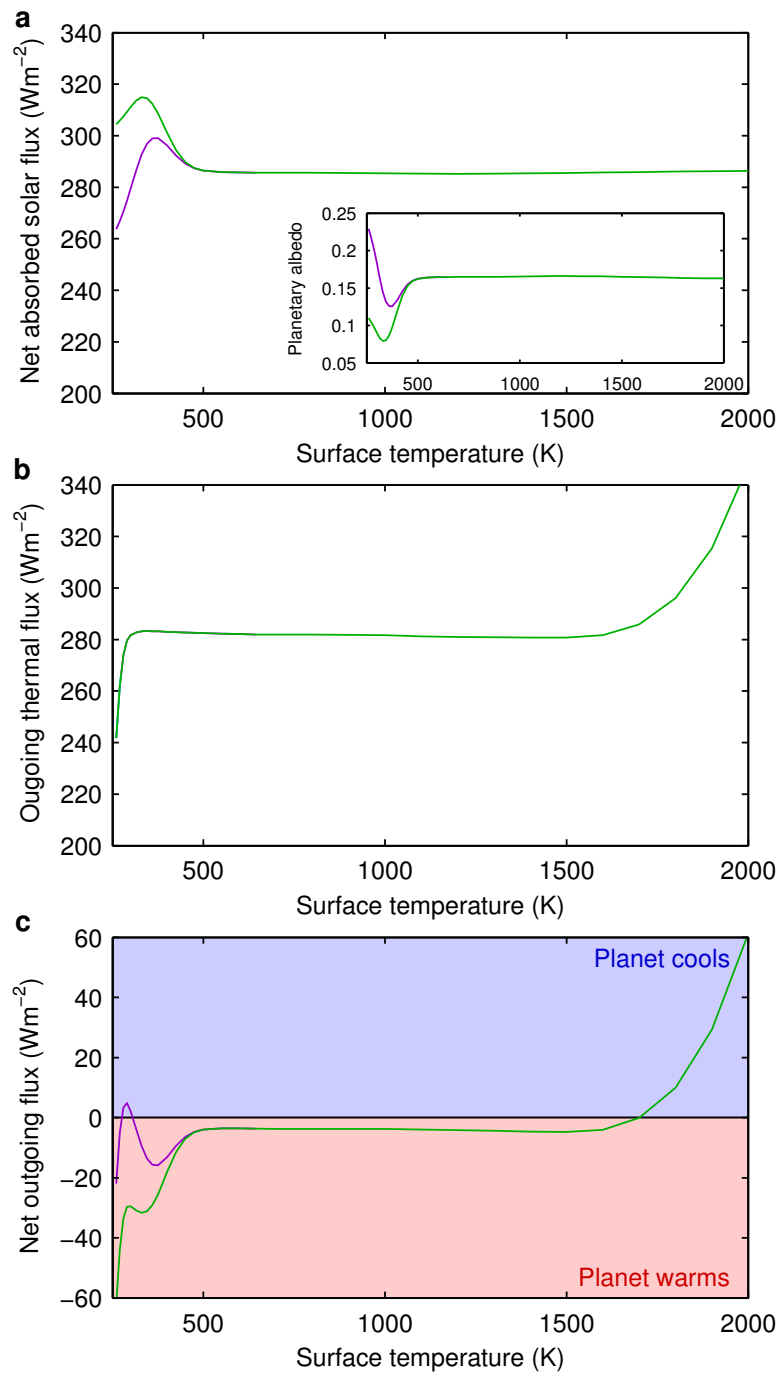

Figure 2: Top of atmosphere fluxes from a pure water atmosphere. Colours identify different surface albedos: green for $12 \%$ and purple for $25 \%$. Steady state climates are found when the net outgoing flux is zero; stable steady states are where the flux is increasing with increasing temperature as it passes through zero and unstable steady states are where it decreases with increasing temperature as it passes through zero. 

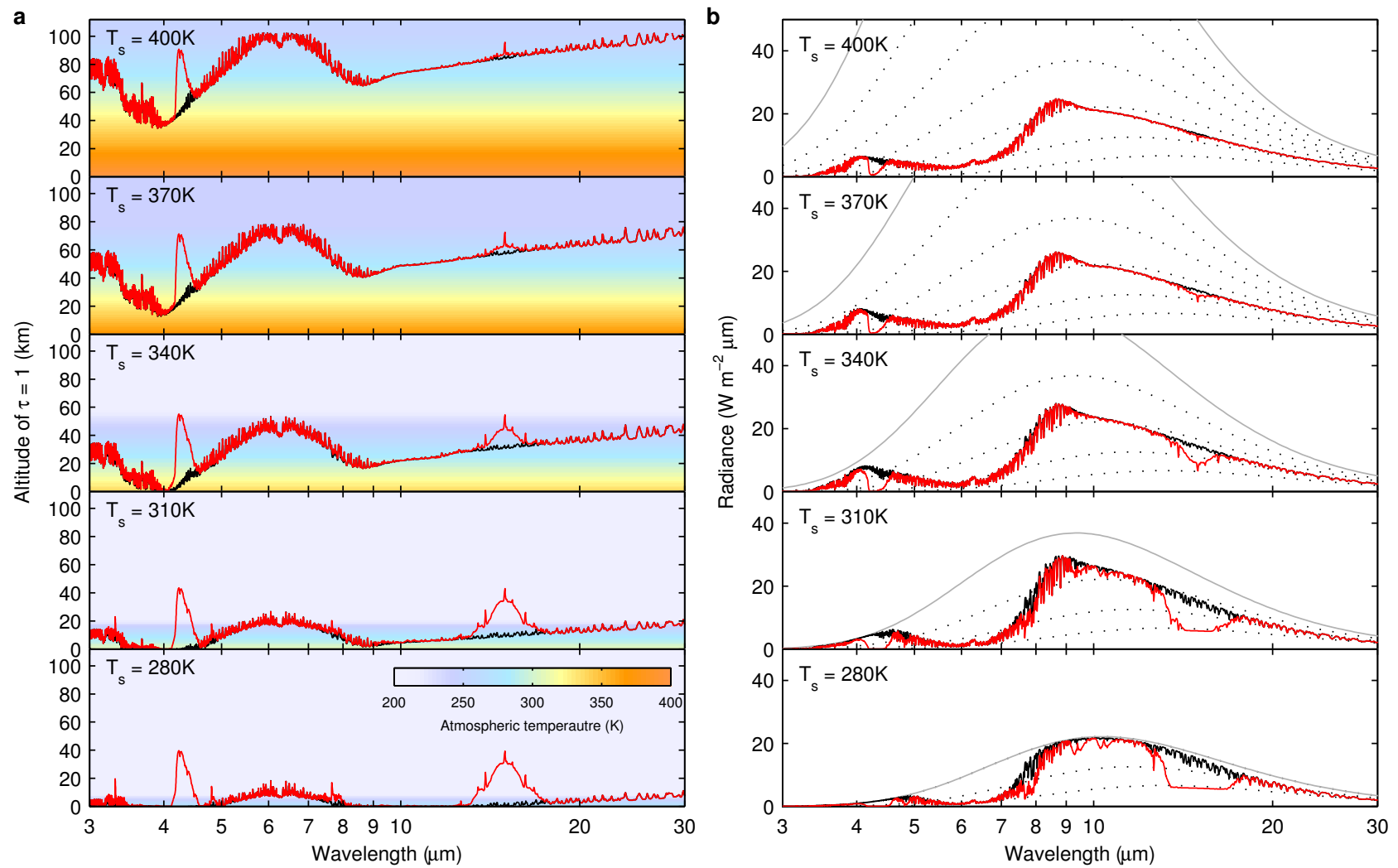

Figure 3: Spectra of thermal emission level and outgoing thermal radiation for transitional atmospheres Black is baseline case, red is with $5000 \mathrm{ppmv} \mathrm{CO}_{2}$. a. Altitude where optical depth is unity. Most outgoing thermal radiation is emitted from this level. Background colour is atmospheric temperature. b. Top of atmosphere emission spectra. Solid grey lines are the surface emission for each case. Dotted grey lines are for Planck functions of $220 \mathrm{~K}, 250 \mathrm{~K}, 280 \mathrm{~K}, 310 \mathrm{~K}, 340 \mathrm{~K}$ and $370 \mathrm{~K}$ up to the surface temperature. The broad 5 to $8 \mu \mathrm{m}$ absorption feature is from water and the smaller absorption features at $4.2 \mu \mathrm{m}$ and $15 \mu \mathrm{m}$ are from carbon dioxide. 

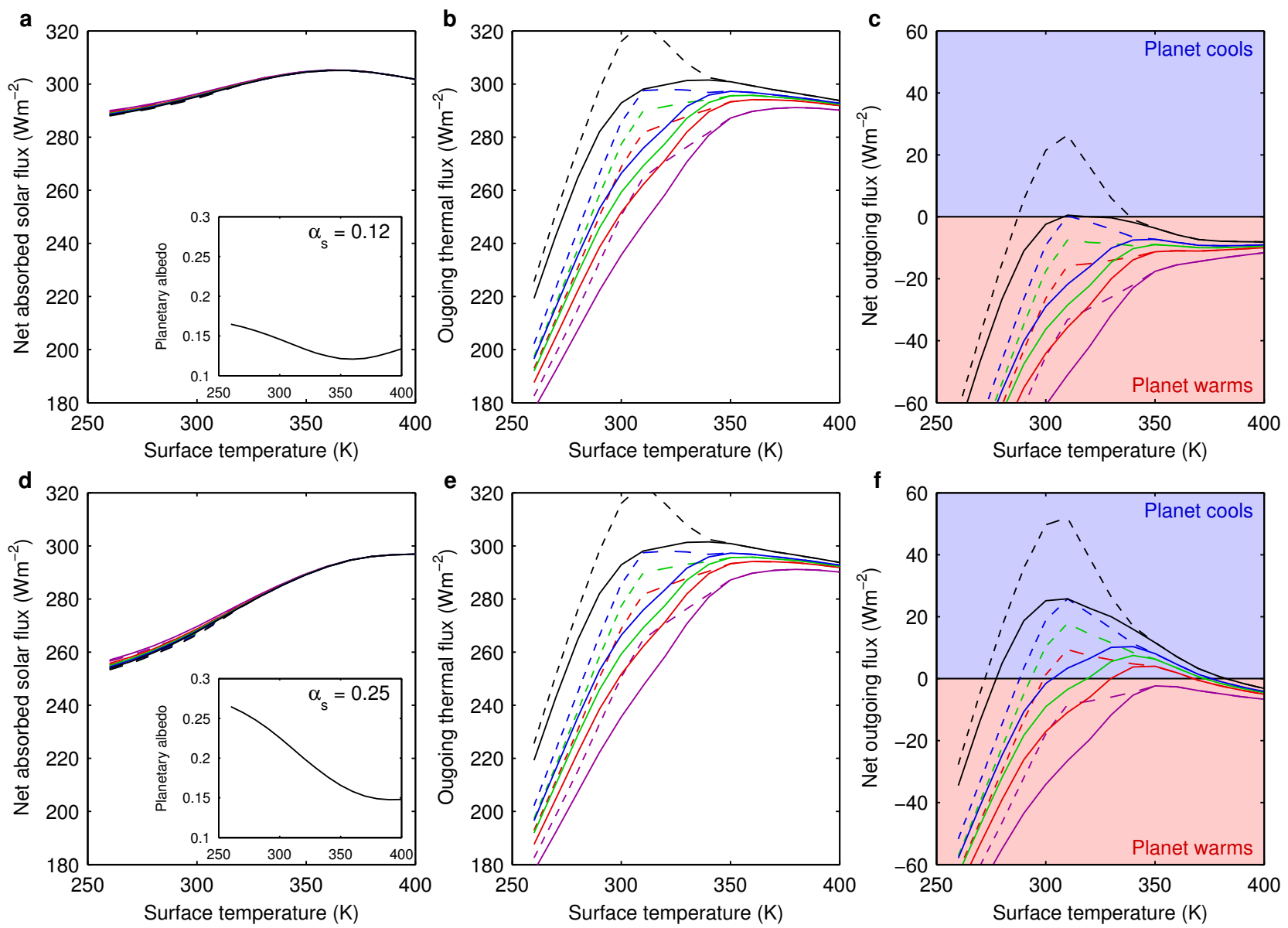

Figure 4: Top of atmosphere fluxes from transitional atmospheres. a-c are with a surface albedo of $12 \%$. d-f are with a surface albedo of $25 \%$. Colours identify the greenhouse gas inventories from Table 1 (black is baseline, blue is preindustrial, green is RCP 8.5 at 2100, red is extreme anthropogenic and purple is arbitrarily high). Solid lines are atmospheres saturated with water vapour throughout whereas dashed lines are subsaturated where the saturation mixing ratio is less than $5 \%$. Steady state climates exist where the net outgoing flux is zero, stable where flux increases with temperature, unstable where flux decreases with temperature. 

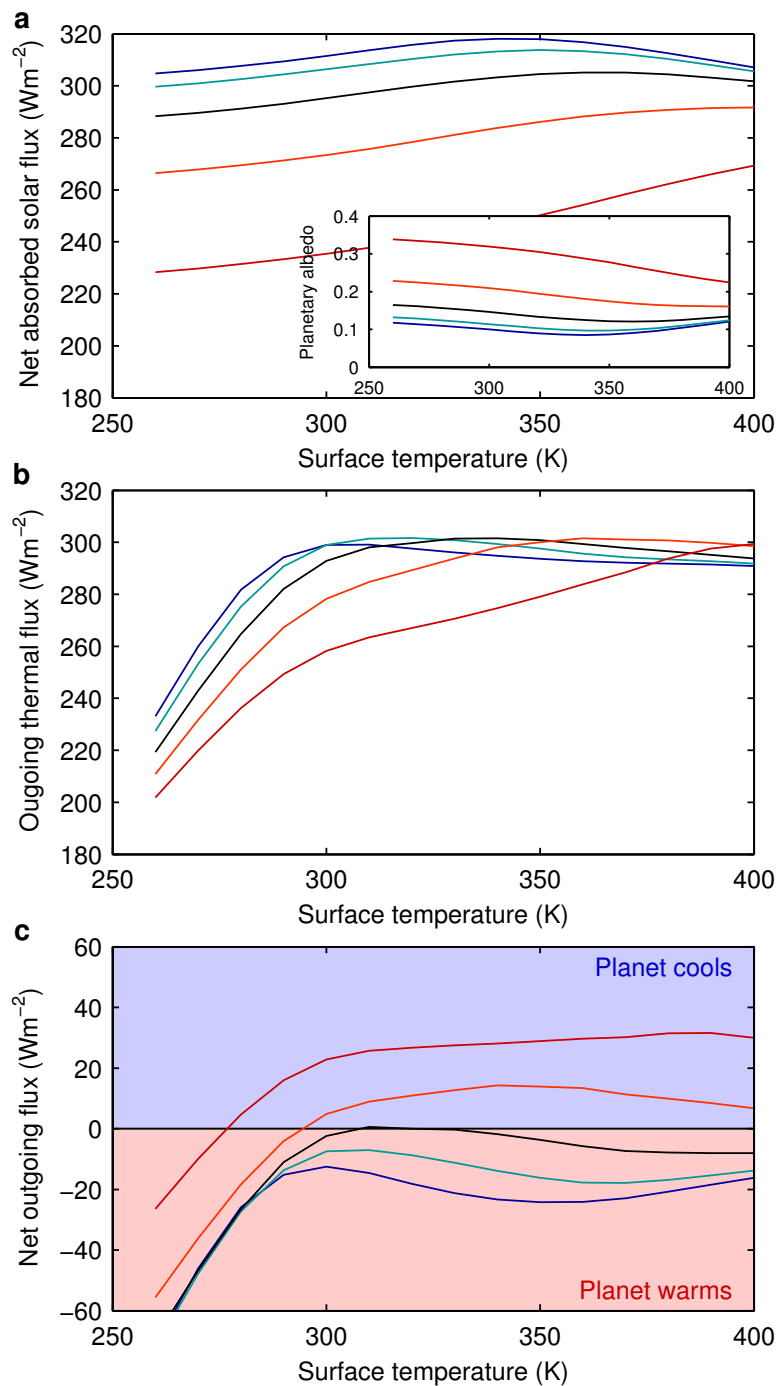

Figure 5: Top of atmosphere fluxes from an ideal gas atmosphere with a varying amount of background gas (nitrogen). All runs for baseline (no non-condensible greenhouse gas) with surface albedo of $12 \%$. Colours are: blue for 0.1 bar, turquoise for 0.33 bar, black for 1 bar, orange for 3 bar and red for 10 bar. 